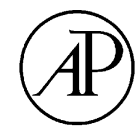

ACADEMIC PRESS

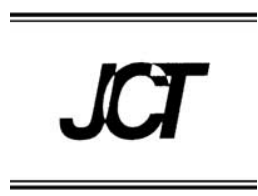

www.elsevier.com/locate/jct

\title{
Low temperature heat capacity of $\mathrm{Nd}_{2} \mathrm{Zr}_{2} \mathrm{O}_{7}$ pyrochlore
}

\author{
S. Lutique a , P. Javorský a , R.J.M. Konings a,*, \\ A.C.G. van Genderen ${ }^{\mathrm{b}}$, J.C. van Miltenburg ${ }^{\mathrm{b}}$, F. Wastin ${ }^{\mathrm{a}}$ \\ a European Commission, Joint Research Centre, Institute for Transuranium Elements, \\ P.O. Box 2340, 76125 Karlsruhe, Germany \\ ${ }^{\mathrm{b}}$ Chemical Thermodynamics Group, Utrecht University, Padualaan 8, 3584 CH, Utrecht, Netherlands
}

Received 28 October 2002; accepted 4 February 2003

\begin{abstract}
Heat capacity of neodymium zirconate $\left(\mathrm{Nd}_{2} \mathrm{Zr}_{2} \mathrm{O}_{7}\right)$ with pyrochlore structure was measured by adiabatic calorimetry and the hybrid adiabatic relaxation method in the temperature range ( 0.45 to 400$) \mathrm{K}$. Its excess component was obtained by comparison with the heat capacity of the lanthanum zirconate. A thermal anomaly was observed below $T=7.2 \mathrm{~K}$. From the heat capacity measurements, the thermodynamic functions of $\mathrm{Nd}_{2} \mathrm{Zr}_{2} \mathrm{O}_{7}$ were determined. (c) 2003 Elsevier Science Ltd. All rights reserved.
\end{abstract}

Keywords: Pyrochlore; Neodymium zirconate; Heat capacity; Thermodynamic properties

\section{Introduction}

Pyrochlores with the general formula $\mathrm{A}_{2} \mathrm{~B}_{2} \mathrm{O}_{7}$ have many potential technological applications because of their refractory nature and their interesting electronic properties, which vary from insulating through semiconductor to metal-like depending on the chemical composition [1]. Lanthanide pyrochlores, in which the light lanthanide elements (La to $\mathrm{Gd}$ ) can occupy the A position, are well known for their magnetic behaviour at low temperature and their fluorescent phosphorescent behaviour [1].

\footnotetext{
${ }^{*}$ Corresponding author. Tel.: +49-7247-951-391; fax: +49-7247-951-566.

E-mail address: konings@itu.fzk.de (R.J.M. Konings).
} 
In nuclear technology there has been a lot of attention paid to zirconate and titanate pyrochlores $(\mathrm{B}=\mathrm{Ti}$ or $\mathrm{Zr})$ and related compounds like zirconolite because of their capability to incorporate not only lanthanides but also actinides. Such compounds have been proposed for plutonium or the minor actinides [2,3] as their natural analogues have shown to be very durable over very long time periods. The thermodynamic stability is a key parameter for modeling the durability and knowledge of the fundamental thermodynamic properties is thus essential.

Only a few studies of the thermodynamic properties of the lanthanide zirconate pyrochlores have been reported. Korneev et al. [4] measured the molar enthalpies of formation of the series of $\mathrm{La}_{2} \mathrm{Zr}_{2} \mathrm{O}_{7}, \mathrm{Pr}_{2} \mathrm{Zr}_{2} \mathrm{O}_{7}, \mathrm{Nd}_{2} \mathrm{Zr}_{2} \mathrm{O}_{7}$, and $\mathrm{Sm}_{2} \mathrm{Zr}_{2} \mathrm{O}_{7}$, Bolech et al. [5-7] the molar enthalpy of formation of $\mathrm{La}_{2} \mathrm{Zr}_{2} \mathrm{O}_{7}$, and Helean et al. [8] of $\mathrm{Gd}_{2} \mathrm{Zr}_{2} \mathrm{O}_{7}$. The heat capacity of $\mathrm{La}_{2} \mathrm{Zr}_{2} \mathrm{O}_{7}$ and $\mathrm{Ce}_{2} \mathrm{Zr}_{2} \mathrm{O}_{7}$ \{in the temperature range (5 to 900) K \} was studied by Bolech et al. [5,7] and in a recent paper we have reported the high-temperature heat capacity $(T=300 \mathrm{~K}$ to $T=1600 \mathrm{~K})$ of $\mathrm{Nd}_{2} \mathrm{Zr}_{2} \mathrm{O}_{7}$ [9]. The present paper extends our work with low temperature measurements in the temperature range $(0.45$ to 400$) \mathrm{K}$.

\section{Experimental}

Neodymium zirconate was obtained by co-precipitation from a solution of zirconium oxi-chloride $\mathrm{ZrOCl}_{2} \cdot 8 \mathrm{H}_{2} \mathrm{O}$ and neodymium nitrate $\mathrm{Nd}\left(\mathrm{NO}_{3}\right)_{3} \cdot 6 \mathrm{H}_{2} \mathrm{O}$ (Alfa Aesar) of stoichiometric concentration which was adjusted to $\mathrm{pH} 11$ by addition of ammonia. The powder thus obtained was sintered for $72 \mathrm{~h}$ at $T=1923 \mathrm{~K}$ in air. Pellets were obtained by pressing calcined beads prepared by the sol-gel method, followed by sintering, as described in detail previously [9]. The pellet density was $97 \%$ of the theoretical density $\left(6325 \mathrm{~kg} \cdot \mathrm{m}^{-3}\right)$. X-ray analysis proved the products to be pure, revealing a cubic pyrochlore phase ( $\mathrm{Fd} 3 \mathrm{~m}$ space group) with lattice parameter $a$ of $1.07 \mathrm{~nm}$.

Two different techniques were used in this study. Adiabatic calorimetry was used to measure the heat capacity of $\mathrm{Nd}_{2} \mathrm{Zr}_{2} \mathrm{O}_{7}$ powder in the temperature range of ( 3 to 400) $\mathrm{K}$ at Utrecht University. The apparatus (laboratory designation CAL V) and its calibration have been described previously [10]. Nine runs were made with this instrument and the results are presented in table 1. Measurements between $T=0.45 \mathrm{~K}$ and $T=27 \mathrm{~K}$ were made by a PPMS instrument (Quantum Design) at ITU using a hybrid adiabatic relaxation method. The apparatus was calibrated using NBS gold. Polycrystalline samples (pellet pieces) with the mass of $15 \mathrm{mg}$ and $1.7 \mathrm{mg}$ were used. The two-tau relaxation method was used to determine the heat capacity with PPMS software. The results of two runs are given in table 2.

\section{Results and discussion}

The results of the different runs by adiabatic calorimetry are in good agreement in the temperature range $(10$ to 400$) \mathrm{K}$, as is shown in figure 1 . At the low temperature 
TABLE 1

Experimental heat capacity $C_{\mathrm{p}, \mathrm{m}}^{0}$ data for $\mathrm{Nd}_{2} \mathrm{Zr}_{2} \mathrm{O}_{7}$ obtained with the adiabatic calorimeter at temperature $T$

\begin{tabular}{|c|c|c|c|c|c|c|c|}
\hline \multirow[t]{2}{*}{$T / \mathrm{K}$} & \multirow{2}{*}{$\frac{C_{\mathrm{p}, \mathrm{m}}^{0}}{\left(\mathrm{~J} \cdot \mathrm{K}^{-1} \cdot \mathrm{mol}^{-1}\right)}$} & \multirow[t]{2}{*}{$T / \mathrm{K}$} & \multirow{2}{*}{$\frac{C_{\mathrm{p}, \mathrm{m}}^{0}}{\left(\mathrm{~J} \cdot \mathrm{K}^{-1} \cdot \mathrm{mol}^{-1}\right)}$} & \multirow[t]{2}{*}{$T / \mathrm{K}$} & \multirow{2}{*}{$\frac{C_{\mathrm{p}, \mathrm{m}}^{0}}{\left(\mathrm{~J} \cdot \mathrm{K}^{-1} \cdot \mathrm{mol}^{-1}\right)}$} & \multirow[t]{2}{*}{$T / \mathrm{K}$} & \multirow{2}{*}{$\frac{C_{\mathrm{p}, \mathrm{m}}^{0}}{\left(\mathrm{~J} \cdot \mathrm{K}^{-1} \cdot \mathrm{mol}^{-1}\right)}$} \\
\hline & & & & & & & \\
\hline \multicolumn{8}{|l|}{ Run 1} \\
\hline 297.03 & 231.7 & 321.08 & 237.8 & 347.92 & 244.6 & 374.81 & 249.9 \\
\hline 298.15 & 231.1 & 324.06 & 238.6 & 350.90 & 245.2 & 377.80 & 250.4 \\
\hline 300.21 & 231.5 & 327.04 & 239.4 & 353.89 & 245.8 & 380.79 & 251.0 \\
\hline 303.20 & 232.6 & 330.02 & 240.2 & 356.87 & 246.5 & 383.79 & 251.7 \\
\hline 306.18 & 233.2 & 333.01 & 241.0 & 359.86 & 247.0 & 386.79 & 252.3 \\
\hline 309.16 & 234.1 & 335.99 & 241.7 & 362.85 & 247.5 & 389.79 & 252.7 \\
\hline 312.14 & 235.2 & 338.97 & 242.4 & 365.84 & 248.2 & 392.79 & 253.1 \\
\hline 315.12 & 236.2 & 341.95 & 243.2 & 368.82 & 248.9 & 395.79 & 253.7 \\
\hline 318.10 & 237.0 & 344.94 & 243.9 & 371.82 & 249.3 & 398.79 & 254.4 \\
\hline \multicolumn{8}{|l|}{ Run 2} \\
\hline 119.67 & 131.0 & 167.49 & 172.3 & 220.71 & 203.2 & 271.18 & 223.3 \\
\hline 121.32 & 132.5 & 170.44 & 174.3 & 223.67 & 204.6 & 274.16 & 224.4 \\
\hline 123.61 & 134.8 & 173.39 & 176.3 & 226.64 & 206.0 & 277.13 & 225.4 \\
\hline 126.53 & 137.8 & 176.34 & 178.3 & 229.60 & 207.4 & 280.10 & 226.5 \\
\hline 129.44 & 140.6 & 179.28 & 180.2 & 232.57 & 208.7 & 283.08 & 227.6 \\
\hline 132.34 & 143.4 & 182.24 & 182.1 & 235.53 & 210.0 & 286.05 & 228.6 \\
\hline 135.25 & 146.1 & 185.19 & 184.0 & 238.50 & 211.2 & 289.02 & 229.6 \\
\hline 138.17 & 148.8 & 188.14 & 185.6 & 241.46 & 212.5 & 292.00 & 230.7 \\
\hline 141.09 & 151.3 & 191.09 & 187.4 & 244.43 & 213.7 & 294.98 & 231.7 \\
\hline 144.01 & 153.9 & 194.05 & 189.2 & 247.40 & 214.9 & 297.96 & 232.6 \\
\hline 146.94 & 156.4 & 197.01 & 190.9 & 250.36 & 216.1 & 300.93 & 233.3 \\
\hline 149.87 & 158.9 & 199.97 & 192.5 & 253.33 & 216.9 & 303.92 & 234.2 \\
\hline 152.80 & 161.2 & 202.93 & 194.2 & 256.31 & 217.7 & 306.90 & 235.2 \\
\hline 155.73 & 163.5 & 205.89 & 195.7 & 259.29 & 219.0 & 309.88 & 236.0 \\
\hline 158.66 & 166.3 & 211.81 & 198.8 & 262.26 & 220.0 & & \\
\hline 161.60 & 168.3 & 214.77 & 200.3 & 265.24 & 221.2 & & \\
\hline 164.55 & 170.2 & 217.74 & 201.7 & 268.21 & 222.3 & & \\
\hline \multicolumn{8}{|l|}{ Run 3} \\
\hline 5.36 & 1.13 & 11.44 & 0.887 & 17.44 & 3.16 & 24.40 & 8.62 \\
\hline 6.58 & 1.215 & 13.49 & 1.35 & 19.64 & 4.37 & 26.95 & 11.20 \\
\hline 8.78 & 0.840 & 15.35 & 1.95 & 21.96 & 6.31 & 29.7 & 13.90 \\
\hline \multicolumn{8}{|l|}{ Run 4} \\
\hline 4.95 & 1.32 & 11.9 & 0.777 & 18.02 & 3.33 & 24.5 & 8.66 \\
\hline 6.45 & 0.496 & 13.15 & 1.32 & 19.29 & 4.29 & 25.85 & 10.1 \\
\hline 8.21 & 0.581 & 14.36 & 1.52 & 20.55 & 5.16 & 27.24 & 11.5 \\
\hline 9.33 & 0.952 & 15.54 & 1.97 & 21.84 & 6.12 & 28.69 & 12.7 \\
\hline 10.54 & 0.691 & 16.77 & 2.73 & 23.17 & 7.23 & 30.17 & 14.3 \\
\hline \multicolumn{8}{|l|}{ Run 5} \\
\hline 3.10 & 0.197 & 5.98 & 0.586 & 8.34 & 0.558 & 10.9 & 0.59 \\
\hline 3.68 & 0.686 & 6.3 & 0.554 & 9.13 & 0.57 & 11.83 & 0.8 \\
\hline 4.03 & 0.645 & 7.37 & 0.499 & 9.97 & 0.592 & 12.77 & 0.89 \\
\hline \multicolumn{8}{|l|}{ Run 6} \\
\hline 3.65 & 0.937 & 7.71 & 0.498 & 10.35 & 0.614 & 13.21 & 1.15 \\
\hline 6.5 & 0.525 & 8.67 & 0.562 & 11.28 & 0.661 & 14.1 & 3.09 \\
\hline 6.81 & 0.462 & 9.47 & 0.558 & 12.23 & 0.826 & & \\
\hline
\end{tabular}


TABLE 1 (continued)

\begin{tabular}{|c|c|c|c|c|c|c|c|}
\hline \multirow[t]{2}{*}{$T / \mathrm{K}$} & \multirow{2}{*}{$\frac{C_{\mathrm{p}, \mathrm{m}}^{0}}{\left(\mathrm{~J} \cdot \mathrm{K}^{-1} \cdot \mathrm{mol}^{-1}\right)}$} & \multirow[t]{2}{*}{$T / \mathrm{K}$} & \multirow{2}{*}{$\frac{C_{\mathrm{p}, \mathrm{m}}^{0}}{\left(\mathrm{~J} \cdot \mathrm{K}^{-1} \cdot \mathrm{mol}^{-1}\right)}$} & \multirow[t]{2}{*}{$T / \mathrm{K}$} & \multirow{2}{*}{$\frac{C_{\mathrm{p}, \mathrm{m}}^{0}}{\left(\mathrm{~J} \cdot \mathrm{K}^{-1} \cdot \mathrm{mol}^{-1}\right)}$} & \multirow[t]{2}{*}{$T / \mathrm{K}$} & \multirow{2}{*}{$\frac{C_{\mathrm{p}, \mathrm{m}}^{0}}{\left(\mathrm{~J} \cdot \mathrm{K}^{-1} \cdot \mathrm{mol}^{-1}\right)}$} \\
\hline & & & & & & & \\
\hline \multicolumn{8}{|l|}{ Run 7} \\
\hline 16.08 & 1.92 & 35.16 & 20.9 & 53.92 & 46.3 & 74.16 & 75.2 \\
\hline 19.38 & 3.96 & 36.78 & 22.8 & 55.71 & 49.1 & 76.04 & 77.7 \\
\hline 21.42 & 5.69 & 38.42 & 24.6 & 57.51 & 51.7 & 77.94 & 80.3 \\
\hline 22.98 & 7.07 & 40.09 & 27.0 & 59.33 & 54.3 & 79.83 & 82.9 \\
\hline 24.42 & 8.35 & 41.76 & 29.3 & 61.15 & 56.9 & 81.74 & 85.5 \\
\hline 25.90 & 9.98 & 43.45 & 31.6 & 62.98 & 59.5 & 83.64 & 88.0 \\
\hline 27.42 & 11.3 & 45.16 & 34.0 & 64.83 & 62.1 & 85.55 & 90.5 \\
\hline 28.93 & 13.1 & 46.88 & 36.4 & 66.68 & 64.7 & 87.47 & 92.9 \\
\hline 30.47 & 14.6 & 48.62 & 38.8 & 68.54 & 67.2 & 89.39 & 95.5 \\
\hline 32.02 & 16.7 & 50.37 & 41.3 & 70.40 & 69.9 & & \\
\hline 33.58 & 18.8 & 52.14 & 43.8 & 72.28 & 72.6 & & \\
\hline \multicolumn{8}{|l|}{ Run 8} \\
\hline 70.18 & 69.9 & 117.92 & 128.9 & 166.86 & 171.8 & 216.23 & 201.1 \\
\hline 72.15 & 72.3 & 120.35 & 131.4 & 169.33 & 173.5 & 218.70 & 202.3 \\
\hline 75.20 & 76.5 & 122.78 & 134.0 & 171.79 & 175.1 & 221.18 & 203.5 \\
\hline 77.48 & 79.7 & 125.21 & 136.4 & 174.25 & 176.7 & 223.66 & 204.6 \\
\hline 79.78 & 82.8 & 127.65 & 138.9 & 176.71 & 178.5 & 226.14 & 205.8 \\
\hline 82.11 & 86.0 & 130.09 & 141.2 & 179.18 & 180.1 & 228.61 & 206.9 \\
\hline 84.45 & 88.9 & 132.53 & 143.5 & 181.64 & 181.7 & 231.09 & 208.0 \\
\hline 86.80 & 92.1 & 134.97 & 145.7 & 184.11 & 183.3 & 233.57 & 209.0 \\
\hline 89.15 & 95.1 & 137.42 & 148.0 & 186.58 & 184.8 & 236.05 & 210.2 \\
\hline 91.52 & 98.1 & 139.86 & 150.2 & 189.05 & 186.3 & 238.52 & 211.3 \\
\hline 93.89 & 101.1 & 142.32 & 152.4 & 191.52 & 187.6 & 241.00 & 212.3 \\
\hline 96.27 & 104.0 & 144.77 & 154.5 & 193.99 & 189.1 & 243.48 & 213.3 \\
\hline 98.66 & 106.9 & 147.22 & 156.6 & 196.46 & 190.6 & 245.96 & 214.4 \\
\hline 101.05 & 109.3 & 149.68 & 158.7 & 198.93 & 192.0 & 248.44 & 215.3 \\
\hline 103.44 & 112.9 & 152.13 & 160.7 & 201.40 & 193.3 & 250.92 & 216.3 \\
\hline 105.85 & 115.6 & 154.58 & 162.6 & 203.87 & 194.7 & 253.40 & 216.9 \\
\hline 108.25 & 118.4 & 157.03 & 164.8 & 206.34 & 196.1 & 255.88 & 217.4 \\
\hline 110.66 & 121.1 & 159.49 & 166.8 & 208.81 & 197.4 & 258.37 & 218.6 \\
\hline 113.08 & 123.7 & 161.94 & 168.5 & 211.28 & 198.7 & 260.85 & 219.6 \\
\hline 115.50 & 126.3 & 164.40 & 170.0 & 213.75 & 199.9 & & \\
\hline \multicolumn{8}{|l|}{ Run 9} \\
\hline 262.50 & 220.0 & 298.46 & 232.7 & 335.64 & 242.3 & 372.91 & 250.0 \\
\hline 263.89 & 220.6 & 300.95 & 232.6 & 338.12 & 243.0 & 375.40 & 250.6 \\
\hline 266.37 & 221.6 & 303.46 & 231.9 & 340.60 & 243.3 & 377.89 & 251.0 \\
\hline 268.83 & 222.2 & 305.94 & 234.6 & 343.08 & 243.9 & 380.38 & 251.3 \\
\hline 271.30 & 223.2 & 308.41 & 235.2 & 345.57 & 244.6 & 382.87 & 251.9 \\
\hline 273.77 & 224.1 & 310.88 & 235.9 & 348.05 & 245.2 & 385.37 & 252.3 \\
\hline 276.23 & 225.1 & 313.35 & 236.5 & 350.53 & 245.6 & 387.86 & 252.8 \\
\hline 278.70 & 225.8 & 315.83 & 237.3 & 353.01 & 246.2 & 390.36 & 253.5 \\
\hline 281.17 & 226.9 & 318.30 & 238.0 & 355.50 & 246.6 & 392.86 & 253.8 \\
\hline 283.64 & 227.8 & 320.77 & 238.5 & 357.98 & 247.2 & 395.36 & 254.3 \\
\hline 286.11 & 228.9 & 323.25 & 239.1 & 360.47 & 247.6 & 397.86 & 254.7 \\
\hline 288.57 & 229.5 & 325.73 & 239.8 & 362.95 & 248.1 & 400.37 & 255.1 \\
\hline 291.05 & 230.3 & 328.20 & 240.7 & 365.44 & 248.7 & & \\
\hline 293.52 & 231.0 & 330.68 & 241.3 & 367.93 & 249.3 & & \\
\hline 295.99 & 232.0 & 333.16 & 241.6 & 370.42 & 249.6 & & \\
\hline
\end{tabular}


TABLE 2

Experimental heat capacity $C_{\mathrm{p}, \mathrm{m}}^{0}$ data for $\mathrm{Nd}_{2} \mathrm{Zr}_{2} \mathrm{O}_{7}$ obtained with the hybrid adiabatic relaxation method at temperature $T$

\begin{tabular}{|c|c|c|c|c|c|c|c|}
\hline \multirow[t]{2}{*}{$T / \mathrm{K}$} & \multirow{2}{*}{$\frac{C_{\mathrm{p}, \mathrm{m}}^{0}}{\left(\mathrm{~J} \cdot \mathrm{K}^{-1} \cdot \mathrm{mol}^{-1}\right)}$} & \multirow[t]{2}{*}{$T / \mathrm{K}$} & \multirow{2}{*}{$\frac{C_{\mathrm{p}, \mathrm{m}}^{0}}{\left(\mathrm{~J} \cdot \mathrm{K}^{-1} \cdot \mathrm{mol}^{-1}\right)}$} & \multirow[t]{2}{*}{$T / \mathrm{K}$} & \multirow{2}{*}{$\frac{C_{\mathrm{p}, \mathrm{m}}^{0}}{\left(\mathrm{~J} \cdot \mathrm{K}^{-1} \cdot \mathrm{mol}^{-1}\right)}$} & \multirow[t]{2}{*}{$T / \mathrm{K}$} & \multirow{2}{*}{$\frac{C_{\mathrm{p}, \mathrm{m}}^{0}}{\left(\mathrm{~J} \cdot \mathrm{K}^{-1} \cdot \mathrm{mol}^{-1}\right)}$} \\
\hline & & & & & & & \\
\hline \multicolumn{8}{|l|}{ Run 1} \\
\hline 1.884 & 1.234 & 3.664 & 0.486 & 5.772 & 0.256 & 9.644 & 0.316 \\
\hline 1.931 & 1.198 & 3.712 & 0.470 & 5.875 & 0.250 & 9.745 & 0.325 \\
\hline 1.977 & 1.164 & 3.757 & 0.461 & 5.976 & 0.246 & 9.847 & 0.334 \\
\hline 2.025 & 1.132 & 3.804 & 0.451 & 6.078 & 0.242 & 9.949 & 0.344 \\
\hline 2.073 & 1.099 & 3.848 & 0.447 & 6.182 & 0.239 & 10.051 & 0.354 \\
\hline 2.119 & 1.069 & 3.897 & 0.437 & 6.282 & 0.236 & 10.153 & 0.365 \\
\hline 2.166 & 1.037 & 3.944 & 0.427 & 6.385 & 0.233 & 10.165 & 0.367 \\
\hline 2.214 & 1.008 & 3.988 & 0.419 & 6.488 & 0.230 & 10.668 & 0.428 \\
\hline 2.260 & 0.982 & 4.038 & 0.412 & 6.591 & 0.228 & 11.184 & 0.504 \\
\hline 2.308 & 0.958 & 4.082 & 0.407 & 6.692 & 0.226 & 11.700 & 0.596 \\
\hline 2.355 & 0.934 & 4.129 & 0.397 & 6.794 & 0.224 & 12.220 & 0.706 \\
\hline 2.402 & 0.909 & 4.175 & 0.393 & 6.895 & 0.223 & 12.741 & 0.832 \\
\hline 2.449 & 0.887 & 4.221 & 0.386 & 6.998 & 0.222 & 13.261 & 0.978 \\
\hline 2.495 & 0.863 & 4.270 & 0.377 & 7.094 & 0.222 & 13.792 & 1.147 \\
\hline 2.542 & 0.842 & 4.314 & 0.373 & 7.194 & 0.221 & 14.299 & 1.325 \\
\hline 2.590 & 0.818 & 4.361 & 0.369 & 7.298 & 0.221 & 14.816 & 1.529 \\
\hline 2.635 & 0.797 & 4.407 & 0.361 & 7.398 & 0.222 & 15.341 & 1.754 \\
\hline 2.683 & 0.778 & 4.452 & 0.360 & 7.501 & 0.222 & 15.882 & 2.000 \\
\hline 2.729 & 0.756 & 4.499 & 0.353 & 7.603 & 0.223 & 16.414 & 2.271 \\
\hline 2.775 & 0.738 & 4.546 & 0.348 & 7.707 & 0.224 & 16.926 & 2.540 \\
\hline 2.821 & 0.721 & 4.593 & 0.341 & 7.808 & 0.226 & 17.444 & 2.829 \\
\hline 2.868 & 0.704 & 4.637 & 0.338 & 7.911 & 0.228 & 17.966 & 3.135 \\
\hline 2.914 & 0.682 & 4.685 & 0.335 & 8.013 & 0.230 & 18.493 & 3.465 \\
\hline 2.960 & 0.665 & 4.731 & 0.331 & 8.114 & 0.232 & 19.019 & 3.803 \\
\hline 3.015 & 0.653 & 4.776 & 0.326 & 8.217 & 0.235 & 19.537 & 4.196 \\
\hline 3.060 & 0.634 & 4.822 & 0.320 & 8.318 & 0.237 & 20.059 & 4.578 \\
\hline 3.107 & 0.619 & 4.872 & 0.315 & 8.421 & 0.241 & 20.591 & 4.989 \\
\hline 3.154 & 0.602 & 4.918 & 0.311 & 8.527 & 0.245 & 21.117 & 5.397 \\
\hline 3.201 & 0.591 & 4.963 & 0.307 & 8.627 & 0.249 & 21.641 & 5.823 \\
\hline 3.248 & 0.572 & 5.010 & 0.302 & 8.730 & 0.254 & 22.155 & 6.259 \\
\hline 3.294 & 0.568 & 5.057 & 0.298 & 8.831 & 0.259 & 22.677 & 6.726 \\
\hline 3.341 & 0.553 & 5.109 & 0.295 & 8.933 & 0.265 & 23.236 & 7.242 \\
\hline 3.386 & 0.549 & 5.161 & 0.290 & 9.034 & 0.271 & 23.735 & 7.699 \\
\hline 3.433 & 0.534 & 5.262 & 0.284 & 9.137 & 0.277 & 24.234 & 8.194 \\
\hline 3.478 & 0.514 & 5.365 & 0.277 & 9.238 & 0.284 & 24.770 & 8.705 \\
\hline 3.525 & 0.513 & 5.467 & 0.270 & 9.339 & 0.291 & 25.278 & 9.229 \\
\hline 3.570 & 0.504 & 5.568 & 0.265 & 9.440 & 0.299 & 25.823 & 9.800 \\
\hline 3.617 & 0.492 & 5.671 & 0.260 & 9.542 & 0.307 & 26.337 & 10.355 \\
\hline \multicolumn{8}{|l|}{ Run 2} \\
\hline 0.465 & 4.277 & 0.611 & 3.419 & 0.914 & 2.539 & 1.420 & 1.686 \\
\hline 0.478 & 4.143 & 0.626 & 3.384 & 0.964 & 2.462 & 1.471 & 1.625 \\
\hline 0.493 & 3.941 & 0.642 & 3.327 & 1.015 & 2.347 & 1.525 & 1.575 \\
\hline 0.508 & 3.982 & 0.656 & 3.282 & 1.066 & 2.238 & 1.571 & 1.529 \\
\hline 0.523 & 3.869 & 0.663 & 3.229 & 1.117 & 2.152 & 1.621 & 1.471 \\
\hline 0.537 & 3.666 & 0.671 & 3.206 & 1.167 & 2.081 & 1.670 & 1.424 \\
\hline 0.552 & 3.717 & 0.713 & 3.054 & 1.217 & 2.008 & 1.721 & 1.381 \\
\hline
\end{tabular}


TABLE 2 (continued)

\begin{tabular}{|c|c|c|c|c|c|c|c|}
\hline \multirow[t]{2}{*}{$T / \mathrm{K}$} & $C_{\mathrm{p}, \mathrm{m}}^{0}$ & \multirow[t]{2}{*}{$T / \mathrm{K}$} & \multirow{2}{*}{$\frac{C_{\mathrm{p}, \mathrm{m}}^{0}}{\left(\mathrm{~J} \cdot \mathrm{K}^{-1} \cdot \mathrm{mol}^{-1}\right)}$} & \multirow[t]{2}{*}{$T / \mathrm{K}$} & \multirow{2}{*}{$\frac{C_{\mathrm{p}, \mathrm{m}}^{0}}{\left(\mathrm{~J} \cdot \mathrm{K}^{-1} \cdot \mathrm{mol}^{-1}\right)}$} & \multirow[t]{2}{*}{$T / \mathrm{K}$} & \multirow{2}{*}{$\frac{C_{\mathrm{p}, \mathrm{m}}^{0}}{\left(\mathrm{~J} \cdot \mathrm{K}^{-1} \cdot \mathrm{mol}^{-1}\right)}$} \\
\hline & $\overline{\left(\mathrm{J} \cdot \mathrm{K}^{-1} \cdot \mathrm{mol}^{-1}\right)}$ & & & & & & \\
\hline 0.567 & 3.550 & 0.763 & 2.927 & 1.268 & 1.930 & 1.768 & 1.334 \\
\hline 0.583 & 3.483 & 0.814 & 2.768 & 1.318 & 1.859 & 1.832 & 1.276 \\
\hline 0.596 & 3.459 & 0.864 & 2.653 & 1.368 & 1.788 & & \\
\hline
\end{tabular}

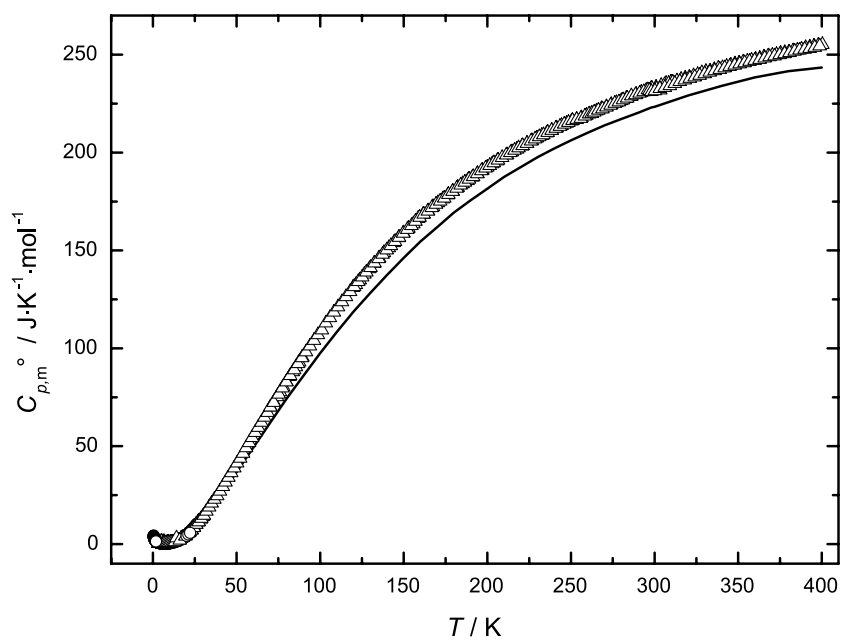

FIGURE 1. Heat capacity $C_{\mathrm{p}, \mathrm{m}}^{0}$ for $\mathrm{Nd}_{2} \mathrm{Zr}_{2} \mathrm{O}_{7} . \Delta$, Adiabatic calorimeter; $\bigcirc$, PPMS; - - reference [5] for $\mathrm{La}_{2} \mathrm{Zr}_{2} \mathrm{O}_{7}$.

limit the data agree well with the results by PPMS whereas above $T=300 \mathrm{~K}$ they join smoothly with the high-temperature heat capacity $(T=400 \mathrm{~K}$ to $T=1500 \mathrm{~K})$ for this compounds measured by DSC [9].

The data obtained by PPMS show that the heat capacity does not continuously decrease with decreasing temperature but that it starts to rise at temperature below $T=7.2 \mathrm{~K}$ indicating the presence of a thermal anomaly shown in figure 2 . Above $T=7.2 \mathrm{~K}$ the data join the adiabatic curve. Below $T=7 \mathrm{~K}$ the adiabatic data are scattered, which is due to the fact that the apparatus was operated at its lower temperature limit.

The Debye temperature was estimated to be $\theta_{\mathrm{D}}=489 \mathrm{~K}$. The heat capacity data below the temperature of $25 \mathrm{~K}$ could be fitted to the equation $C_{\mathrm{p}}^{0}=\alpha T^{3}$, except in the temperature range of the thermal anomaly, yielding $\alpha=5.564 \cdot 10^{-4} \mathrm{~J} \cdot \mathrm{K}^{-4} \cdot \mathrm{mol}^{-1}$. The plot of $C_{\mathrm{p}}^{0} / T$ versus $T^{2}$ below the temperature of $25 \mathrm{~K}$ is shown in figure 3 . At $T=7.2 \mathrm{~K}$, the heat capacity $C_{\mathrm{p}}^{0}\left(\mathrm{Nd}_{2} \mathrm{Zr}_{2} \mathrm{O}_{7}, 7.2 \mathrm{~K}\right)=0.208 \mathrm{~J} \cdot \mathrm{K}^{-1} \cdot \mathrm{mol}^{-1}$.

Figure 1 compares our results for $\mathrm{Nd}_{2} \mathrm{Zr}_{2} \mathrm{O}_{7}$ to the heat capacity of lanthanum zirconate $\mathrm{La}_{2} \mathrm{Zr}_{2} \mathrm{O}_{7}$ obtained by Bolech et al. [5,7] in the temperature range (20 to 400) $\mathrm{K}$ using the same adiabatic calorimeter. The $\mathrm{Nd}_{2} \mathrm{Zr}_{2} \mathrm{O}_{7}$ curve is systematically higher than that of $\mathrm{La}_{2} \mathrm{Zr}_{2} \mathrm{O}_{7}$. This difference corresponds to an excess component 


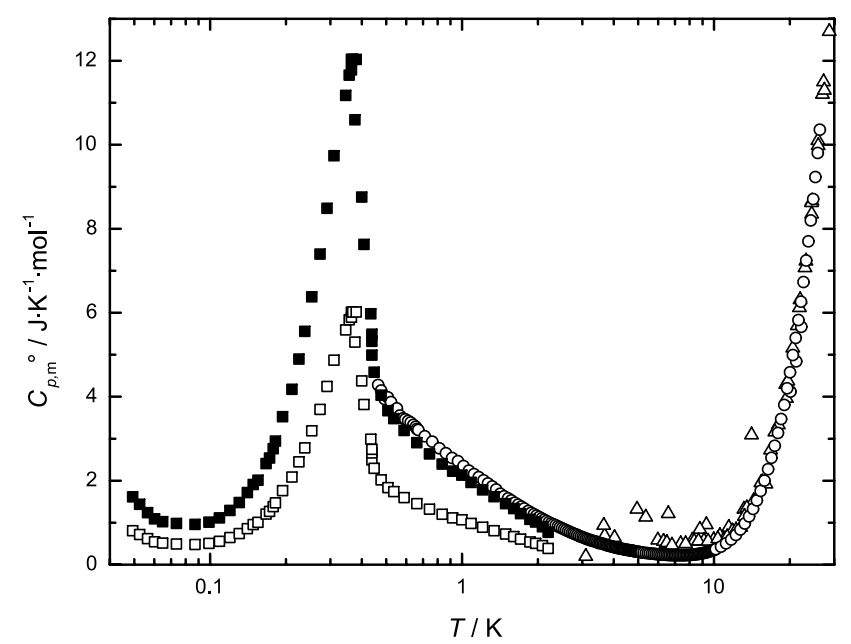

FIGURE 2. Heat capacity $C_{\mathrm{p}, \mathrm{m}}^{0}$ of $\mathrm{Nd}_{2} \mathrm{Zr}_{2} \mathrm{O}_{7}$ between $T=0 \mathrm{~K}$ and $T=25 \mathrm{~K}$. $\triangle$, by Adiabatic calorimeter; $\bigcirc$, PPMS; $\square$, reference [14]; $\mathbf{\square}$, reference [14] corrected for the number of $\mathrm{Nd}^{3+}$ ions in the structure as described in the text.

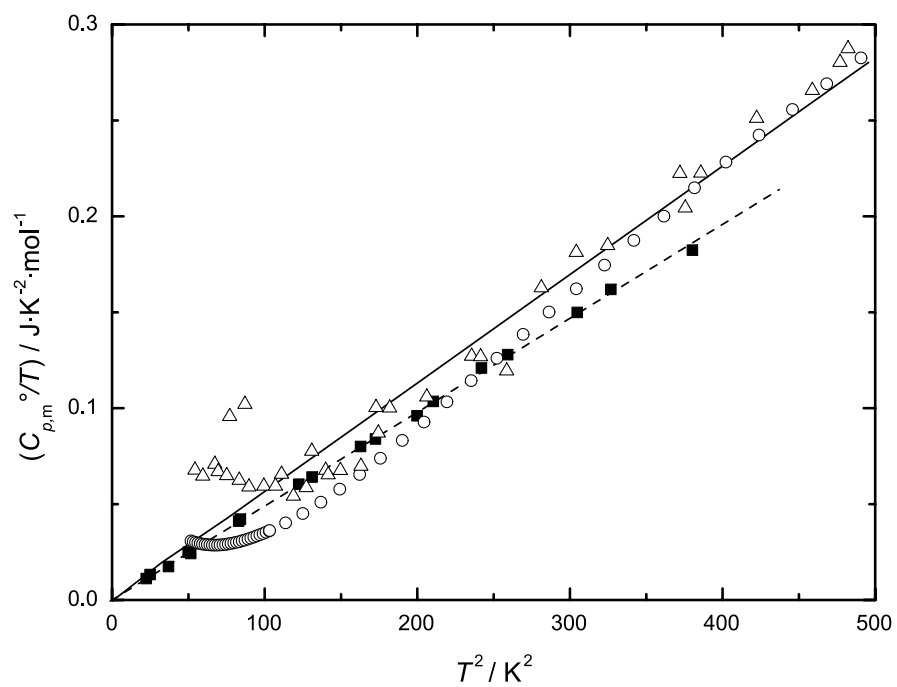

FIGURE 3. Low temperature heat capacity of $C_{\mathrm{p}, \mathrm{m}}^{0} \mathrm{Nd}_{2} \mathrm{Zr}_{2} \mathrm{O}_{7}$. $\Delta$, Adiabatic calorimeter; $\bigcirc$, PPMS; —, $\alpha T^{3}$ fit; $\mathbf{\square}$, reference [5] for $\mathrm{La}_{2} \mathrm{Zr}_{2} \mathrm{O}_{7} ;---, \alpha T^{3}$ fit to the results from reference [5].

to the heat capacity $C_{\text {exs }}$, a so-called Schottky anomaly, that arises from the lowering of the magnetic ordering (splitting of the ground term) by the crystalline electric field (Stark effect) [11]. The $\mathrm{Nd}^{3+}$ ion has a $4 \mathrm{f}^{3}$ electronic configuration and the ground state of the free ion is ${ }^{4} \mathrm{I}_{9 / 2}\left(g_{0}=10\right)$ which in the ionic crystal lattice splits into five 
Kramers doublets $(g=2)$. Lanthanum zirconate has no excess component in this temperature range due to very stable $4 \mathrm{f}^{0}$ electronic configuration of the $\mathrm{La}^{3+}$ ion, and its heat capacity consists only of the lattice component. Thus the total heat capacity $C_{\mathrm{p}}^{0}$ is given by

$$
C_{\mathrm{p}}^{0}=C_{\mathrm{lat}}+C_{\mathrm{exs}},
$$

where $C_{\text {lat }}$ is the lattice contribution to the heat capacity. The excess component of neodymium zirconate can be approximately deduced from the difference with the heat capacity of lanthanum zirconate. The data obtained from $T=20 \mathrm{~K}$ to $T=400 \mathrm{~K}$ reveal the Schottky anomaly to the heat capacity.

The excess component can also be calculated from the crystal field energies according the equation [12]

$$
C_{\text {exs }}=Q^{-2} R^{-1} T^{-2}\left[Q \sum_{i=1}^{n} g_{i} E_{i}^{2} \exp \left(-E_{i} / R T\right)-\left\{\sum_{i=1}^{n} g_{i} E_{i} \exp \left(-E_{i} / R T\right)\right\}^{2}\right],
$$

where $Q$ is the partitioning function described in equation (3) by the MaxwellBoltzmann distribution law, $T$ the temperature, $R$ the universal gas constant, and $E_{i}$ the energy of the level $i$, and $g_{i}$ its degeneracy.

$$
Q=\sum_{i=0}^{n} g_{i} \exp \left(-E_{i} / R T\right) \text {. }
$$

As a first approximation the excess component was calculated from the energy levels of $\mathrm{Nd}^{3+}$ in $\mathrm{Nd}_{2} \mathrm{O}_{3}$ or $\mathrm{NdAlO}_{3}$ [13], as the crystal field splitting in $\mathrm{Nd}_{2} \mathrm{Zr}_{2} \mathrm{O}_{7}$ is not known. However, poor agreement with the experimental values for $\mathrm{Nd}_{2} \mathrm{Zr}_{2} \mathrm{O}_{7}$ was observed, indicating that the crystal field energy of the $\mathrm{Nd}^{3+}$ ion in the pyrochlore structure are significantly different from those in these two compounds.

Figure 2 shows the heat capacity measured by both techniques in the temperature range $(0.45$ to 20$) \mathrm{K}$ using a logarithmic temperature scale. Only the high-temperature tail of an anomaly is present in our results, the remainder of the peak occurs below the lower temperature limit of the PPMS apparatus. This anomaly should be considered as the onset of a cooperative transition to remove the ground state degeneracy of the doublet.

Also shown in the figure are the heat capacity data for the same compound reported by Blöte et al. [14] in the temperature range ( 0.05 to 3$) \mathrm{K}$. These authors observed the maximum in the anomaly at $T=0.37 \mathrm{~K}$, whereas they cite the work of van Geuns who found a Curie temperature around $T=0.6 \mathrm{~K}$ from magnetic susceptibility measurements. Our results are systematically higher than those of Blöte et al. [14], but the interpretation of their results is not fully clear, as we will discuss in the next section.

Similar to the heat capacity, the entropy $S^{0}$ is described as the sum of a lattice component $S_{\text {lat }}$ and an excess component $S_{\text {exs }}[11]$

$$
S^{0}=S_{\text {lat }}+S_{\text {exs }} .
$$


TABLE 3

Thermodynamic functions of $\mathrm{Nd}_{2} \mathrm{Zr}_{2} \mathrm{O}_{7}$ from $T=10 \mathrm{~K}$ to $T=400 \mathrm{~K}\left(\Phi_{\mathrm{m}}^{0}=\left[\Delta G_{\mathrm{m}}^{0}(T)-\Delta H_{\mathrm{m}}^{0}(0)\right] / T\right)$

\begin{tabular}{|c|c|c|c|c|}
\hline \multirow[t]{2}{*}{$T / \mathrm{K}$} & \multirow{2}{*}{$\frac{C_{\mathrm{p}, \mathrm{m}}^{0}}{\left(\mathrm{~J} \cdot \mathrm{K}^{-1} \cdot \mathrm{mol}^{-1}\right)}$} & \multirow{2}{*}{$\frac{S_{\mathrm{m}}^{0}(T)}{\left(\mathrm{J} \cdot \mathrm{K}^{-1} \cdot \mathrm{mol}^{-1}\right)}$} & \multirow{2}{*}{$\frac{\Delta_{298.15}^{T} H_{m}(T)}{\left(\mathrm{J} \cdot \mathrm{mol}^{-1}\right)}$} & \multirow{2}{*}{$\frac{\Phi_{\mathrm{m}}^{0}}{\left(\mathrm{~J} \cdot \mathrm{K}^{-1} \cdot \mathrm{mol}^{-1}\right)}$} \\
\hline & & & & \\
\hline 10 & 0.354 & 12.76 & 8.76 & 11.88 \\
\hline 15 & 1.61 & 13.09 & 13.04 & 12.22 \\
\hline 20 & 4.70 & 13.92 & 27.9 & 12.52 \\
\hline 25 & 9.41 & 15.43 & 62.0 & 12.95 \\
\hline 30 & 14.18 & 17.53 & 120.1 & 13.53 \\
\hline 35 & 20.69 & 20.20 & 206.9 & 14.29 \\
\hline 40 & 26.88 & 23.34 & 325.1 & 15.21 \\
\hline 45 & 33.74 & 26.91 & 476.7 & 16.32 \\
\hline 50 & 40.80 & 30.83 & 663.0 & 17.57 \\
\hline 55 & 47.98 & 35.05 & 884.6 & 18.97 \\
\hline 60 & 55.25 & 39.54 & 1143 & 20.49 \\
\hline 65 & 62.35 & 44.24 & 1437 & 22.13 \\
\hline 70 & 69.29 & 49.12 & 1766 & 23.89 \\
\hline 75 & 76.31 & 54.14 & 2130 & 25.74 \\
\hline 80 & 83.09 & 59.28 & 2529 & 27.67 \\
\hline 85 & 89.68 & 64.52 & 2961 & 29.68 \\
\hline 90 & 96.18 & 69.83 & 3426 & 31.76 \\
\hline 95 & 102.5 & 75.20 & 3922 & 33.92 \\
\hline 100 & 108.2 & 80.60 & 4449 & 36.11 \\
\hline 110 & 120.4 & 91.48 & 5592 & 40.65 \\
\hline 120 & 131.2 & 102.4 & 6850 & 45.34 \\
\hline 130 & 141.1 & 113.3 & 8212 & 50.15 \\
\hline 140 & 150.3 & 124.1 & 9669 & 55.05 \\
\hline 150 & 159.0 & 134.8 & 11215 & 60.01 \\
\hline 160 & 167.2 & 145.3 & 12846 & 65.01 \\
\hline 170 & 174.0 & 155.6 & 14552 & 70.03 \\
\hline 180 & 180.7 & 165.8 & 16326 & 75.07 \\
\hline 190 & 186.8 & 175.7 & 18163 & 80.11 \\
\hline 200 & 192.5 & 185.4 & 20060 & 85.13 \\
\hline 210 & 198.0 & 195.0 & 22012 & 90.13 \\
\hline 220 & 202.9 & 204.3 & 24017 & 95.11 \\
\hline 230 & 207.5 & 213.4 & 26069 & 100.1 \\
\hline 240 & 211.9 & 222.3 & 28166 & 105.0 \\
\hline 250 & 216.0 & 231.1 & 30305 & 109.8 \\
\hline 260 & 219.3 & 239.6 & 32482 & 114.7 \\
\hline 270 & 222.8 & 248.0 & 34692 & 119.4 \\
\hline 280 & 226.4 & 256.1 & 36938 & 124.2 \\
\hline 290 & 229.9 & 264.1 & 39220 & 128.9 \\
\hline 298.15 & 232.7 & 270.5 & 41105 & 132.6 \\
\hline 300 & 233.1 & 272.0 & 41535 & 133.5 \\
\hline 310 & 236.0 & 279.6 & 43881 & 138.1 \\
\hline 320 & 238.4 & 287.2 & 46253 & 142.6 \\
\hline 330 & 241.1 & 294.6 & 48650 & 147.1 \\
\hline 340 & 243.2 & 301.8 & 51072 & 151.6 \\
\hline 350 & 245.5 & 308.9 & 53515 & 156.0 \\
\hline 360 & 247.5 & 315.8 & 55980 & 160.3 \\
\hline 370 & 249.5 & 322.6 & 58465 & 164.6 \\
\hline 380 & 251.3 & 329.3 & 60969 & 168.8 \\
\hline 390 & 253.4 & 335.84 & 63493 & 173.0 \\
\hline
\end{tabular}


For the same reasons as mentioned earlier, the lattice entropy of neodymium zirconate can be represented by the entropy of lanthanum zirconate. The heat capacity of $\mathrm{La}_{2} \mathrm{Zr}_{2} \mathrm{O}_{7}$ was fitted to $C_{\mathrm{p}}^{0}\left(\mathrm{La}_{2} \mathrm{Zr}_{2} \mathrm{O}_{7}\right)=4.895 \cdot 10^{-4}(T / \mathrm{K})^{3}$ in the temperature range $(0$ to 25$) \mathrm{K}$. The value $S^{0}\left(\mathrm{La}_{2} \mathrm{Zr}_{2} \mathrm{O}_{7}, 7.2 \mathrm{~K}\right)=S_{\text {lat }}\left(\mathrm{Nd}_{2} \mathrm{Zr}_{2} \mathrm{O}_{7}, 7.2 \mathrm{~K}\right)$ $=0.0609 \mathrm{~J} \cdot \mathrm{K}^{-1} \cdot \mathrm{mol}^{-1}$ was obtained by integration of $C_{\mathrm{p}}^{0}\left(\mathrm{La}_{2} \mathrm{Zr}_{2} \mathrm{O}_{7}\right) / T$ from $T=0 \mathrm{~K}$ to $T=7.2 \mathrm{~K}$.

The excess component of the entropy can be calculated from the crystal field energies according the equation

$$
S_{\mathrm{exs}}=R \ln \left(g_{0}\right)+R \ln \left\{\sum_{i=1}^{n} g_{i} \exp \left(-E_{i} / R T\right)\right\} .
$$

The first term represents the contribution of the ground state, the second term that of the crystal field energies, which is only relevant at temperatures above $T=10 \mathrm{~K}$ for $\mathrm{Nd}_{2} \mathrm{Zr}_{2} \mathrm{O}_{7}$ (as the first excited state is very probably $>100 \mathrm{~cm}^{-1}$ ). As discussed above, the ground state of the $\mathrm{Nd}^{3+}$ ion in $\mathrm{Nd}_{2} \mathrm{Zr}_{2} \mathrm{O}_{7}$ is a doublet $(g=2)$ and thus $S_{\text {exs }}\left(\mathrm{Nd}_{2} \mathrm{Zr}_{2} \mathrm{O}_{7}, 7.2 \mathrm{~K}\right)=2 R \ln (2)=11.526 \mathrm{~J} \cdot \mathrm{K}^{-1} \cdot \mathrm{mol}^{-1}$.

Blöte et al. [14] reported $\Delta S / R=0.698=1.007 \ln (2)$ for the entropy of the low temperature anomaly in $\mathrm{Nd}_{2} \mathrm{Zr}_{2} \mathrm{O}_{7}$, and argued that this is an indication for a doublet ground state. However, it is a factor 2 lower than the theoretical value, which probably means that they normalised their results to one mole of $\mathrm{Nd}^{3+}$ ions. The heat capacity of $\mathrm{Nd}_{2} \mathrm{Zr}_{2} \mathrm{O}_{7}$ obtained by doubling the experimental values of Blöte et al. is plotted in figure 2, which shows that a very good agreement with the experimental values measured by PPMS is now obtained.

The total molar entropy of $\mathrm{Nd}_{2} \mathrm{Zr}_{2} \mathrm{O}_{7}$ at $T=7.2 \mathrm{~K}$ is the sum of these two terms (lattice and excess components) yielding $S^{0}\left(\mathrm{Nd}_{2} \mathrm{Zr}_{2} \mathrm{O}_{7}, 7.2 \mathrm{~K}\right)=11.587 \mathrm{~J} \cdot \mathrm{K}^{-1} \cdot \mathrm{mol}^{-1}$. Using this value the thermodynamic functions of $\mathrm{Nd}_{2} \mathrm{Zr}_{2} \mathrm{O}_{7}$ in the temperature range (10 to 400$) \mathrm{K}$ were derived, as summarised in table 3 . The value obtained for the heat capacity and the entropy at the standard temperature of $298.15 \mathrm{~K}$ are $C_{\mathrm{p}}^{0}\left(\mathrm{Nd}_{2} \mathrm{Zr}_{2} \mathrm{O}_{7}, 298.15 \mathrm{~K}\right)=(232.7 \pm 0.3) \mathrm{J} \cdot \mathrm{K}^{-1} \cdot \mathrm{mol}^{-1}$ and $S^{0}\left(\mathrm{Nd}_{2} \mathrm{Zr}_{2} \mathrm{O}_{7}, 298.15 \mathrm{~K}\right)=$ $(264.1 \pm 0.6) \mathrm{J} \cdot \mathrm{K}^{-1} \cdot \mathrm{mol}^{-1}$.

\section{Conclusion}

The thermodynamic functions of $\mathrm{Nd}_{2} \mathrm{Zr}_{2} \mathrm{O}_{7}$ pyrochlore were obtained from the low temperature heat capacity measurements. The excess component of the heat capacity was determined by assuming the heat capacity of $\mathrm{La}_{2} \mathrm{Zr}_{2} \mathrm{O}_{7}$ to represent the lattice component of $\mathrm{Nd}_{2} \mathrm{Zr}_{2} \mathrm{O}_{7}$. The resulting values were compared with calculations using the crystal field energies of the $\mathrm{Nd}^{3+}$ ion in $\mathrm{Nd}_{2} \mathrm{O}_{3}$ and $\mathrm{NdAlO}_{3}$. The significant difference between the experimental and calculated data is probably due to two facts: the lattice component is not constant over the lanthanide series, and the energy levels of the $\mathrm{Nd}^{3+}$ are different in the pyrochlore structure. In order to better resolve the lattice and the excess component of the heat capacity for the lanthanide zirconate pyrochlore series, measurements of the heat capacity of some other 
lanthanide zirconates ( $\mathrm{Ln}=\mathrm{Eu}$ and $\mathrm{Gd})$ by the same techniques and the determination of the energy levels of lanthanide ions in the pyrochlore structure by optical spectroscopy are currently in progress and will be discussed in a following paper.

\section{Acknowledgements}

We would like to thank J.G. Boshoven, H. Hein, A. Fernandez, and R. Voet for their assistance for the samples preparation, S.L. and P.J. acknowledge the European Commission for support given in the frame of the program "training and mobility of researchers".

\section{References}

[1] M.A. Subramanian, G. Aramudan, G.V. Subba Rao, Prog. Solid State Chem. 15 (1983) $55-143$.

[2] B.F. Woodfield, J. Boerio-Goates, J.L. Shapiro, R.L. Putnam, A. Navrotsky, J. Chem. Thermodyn. 31 (1999) 245-253.

[3] R.L. Putnam, A. Navrotsky, B.F. Woodfield, J. Boerio-Goates, J.L. Shapiro, J. Chem. Thermodyn. 31 (1999) 229-243.

[4] V.R. Korneev, V.B. Glushkova, E.K. Keler, Izv. Akad. Nauk SSSR, Neorg. Mater. 7 (1971) $886-887$.

[5] M. Bolech, Ph.D. Thesis, University of Amsterdam, Amsterdam, Netherlands, 1998.

[6] M. Bolech, E.H.P. Cordfunke, F.J.J.G. Janssen, A. Navrotsky, J. Am. Ceram. Soc. 78 (1995) 2257-2258.

[7] M. Bolech, E.H.P. Cordfunke, A.C.G. Van Genderen, R.R. Van Der Laan, F.J.J.G. Janssen, J.C. Van Miltenburg, J. Phys. Chem. Solids 58 (1997) 433-439.

[8] K.B. Helean, B.D. Begg, A. Navrotsky, B. Ebbinghaus, W.J. Weber, R.C. Ewing, Mat. Res. Soc. Symp. Proc. 663 (2001) 691-697.

[9] S. Lutique, R.J.M. Konings, V.V. Rondinella, J. Somers, T. Wiss, J. Alloy Compd. 352 (2003) 1-5.

[10] J.C. Van Miltenburg, G.J.K. van den Berg, M.J. van Bommel, J. Chem. Thermodyn. 19 (1987) 1129-1138.

[11] R.J.M. Konings, J. Nucl. Mater. 295 (2001) 57-63.

[12] B.H. Justice, E.F.J. Westrum, J. Phys. Chem. 67 (1963) 339-345.

[13] C.A. Morrison, R.P. Leavitt, in: K.A. Gschneidner, L. Eyring Jr. (Eds.), Handbook of the Physics and Chemistry of Rare Earth, vol. 5, 1982 (Chapter 46).

[14] H.W.J. Blöte, R.F. Wielinga, W.J. Huiskamp, Physica 43 (1969) 549-568.

AG02/025 